\title{
Verletzungen der oberen Extremität im Kindesalter
}

\author{
Mike Oberle, Wolfgang Schlickewei
}

\section{Zusammenfassung}

Verletzungen der oberen Extremität im Wachstumsalter sind relativ häufig. Der größte Anteil fällt dabei auf die distale Unterarmfraktur. Ursachen sind meist Freizeit- oder Sportverletzungen. Das Geburtstrauma spielt bei den Klavikulafrakturen und bei Frakturen im Bereich des proximalen Oberarms eine Rolle. Bei Verletzungen im Kindesalter werden sowohl an die Diagnostik als auch an die Therapie spezielle Anforderungen gestellt. Im Unterschied zu Frakturen im Erwachsenenalter haben Frakturen im Kindesalter durch die offenen Wachstumsfugen spezielle Probleme, aber auch Möglichkeiten zur Spontankorrektur, welche in die Behandlung mit eingeschlossen werden müssen. Zur kindgerechten Behandlung gehören eine rasche Diagnostik, Kenntnis der Grenzen der Spontankorrektur und eine unmittelbare, schmerzfreie und definitive Therapie. Es muss grundsätzlich zwischen intraartikulären und extraartikulären Frakturen unterschieden werden. Während bei intraartikulären Frakturen keine Spontankorrektur eintritt und daher eine anatomische Reposition gefordert wird, kann abhängig von der Lokalisation bei extraartikulären Frakturen die Möglichkeit der Spontankorrektur berücksichtigt werden. Therapie der Wahl ist für Frakturen im Schaftbereich die minimalinvasive, intramedulläre Schienung mit TENS. Im metaphysären und epiphysären Bereich kommen Kirschner-Draht-Osteosynthesen und Schraubenosteosynthesen zum Einsatz. Plattenosteosynthesen kommen nur bei Ausnahmeindikationen zum Einsatz. Im folgenden Beitrag sollen die Diagnostik und Therapie bei Verletzungen der oberen Extremität im Kindesalter im Vordergrund stehen.

\section{Upper Limb Injuries in Childhood}

Lesions of the upper limb in children are quite frequent occurrences. Fractures of the distal forearm account for the main part of them. Most injuries occur during leisure or sports activities. Trauma during birth is often the cause of fractures of the clavicula and of the proximal humeral fractures. Injuries in children require a special kind of diagnostic method and therapy. In contrast to fractures in adults, due to the open epiphyseal cartilage, fractures in children have some particular risks and also specific opportunities for spontaneous remodelling. In particular for children it is necessary to make a sure and rapid diagnosis, to recognise the limits of remodelling and to initiate a definitive therapy which is free of pain. One must differentiate between extraarticular and intra-articular fractures. There is no spontaneous remodelling in intra-articular fractures, so an anatomic reduction in these fractures must be included in the planning. Depending on the location there is a high level of spontaneous remodelling in extra-articular fractures in children. The state of the art in the surgical therapy for diaphyseal fractures is intramedullary nailing with elastic titanium nails. Fractures in the metaphyseal or epiphyseal area are treated with K-wires or screws. Osteosynthesis with plates should only be used for special indications. The present article illustrates particularly the diagnostic processes and therapy for fractures of the upper limb in children.

\section{Einleitung}

Verletzungen des Bewegungsapparats im Kindesalter gehören zu den häufigsten behandlungsbedürftigen Diagnosen in traumatologischen und kindertraumatologischen Abteilungen. Vor allem durch die zunehmende Beliebtheit von sog. Hochrasanzsportarten wie Skateboard, Inlinefahren, Mountainbike und entsprechenden Wintersportarten haben diese Verletzungen in den letzten

OP-JOURNAL 2010; 26: 90-97

(c) Georg Thieme Verlag KG Stuttgart · New York DOI http://dx.doi.org/10.1055/s-0030-1250248
Jahren eine deutliche Steigerung erfahren. Moderne, minimalinvasive Operationsmethoden haben dazu beigetragen, dass diese Verletzungen kindgerecht und adäquat behandelt werden können. Dennoch stellen z.B. Verletzungen des kindlichen Ellenbogens auch heute noch oft eine Herausforderung für den Operateur dar, sodass eine kindertraumatologische Expertise gefordert werden sollte [1].

\section{Klavikulafraktur}

Häufigste geburtstraumatische Schädigung. Im Kindesalter macht die Klavikulafraktur 15\% aller Knochenbrüche aus. Ursache ist meist ein direktes Trauma.
Bei kleineren Kindern wird die Fraktur durch den dicken Periostschlauch geschient und ist meist undisloziert.

\section{Klinische Diagnostik}

- Druckschmerz und Schmerzen bei Abduktion des Armes

- Klaviertastenphänomen (v.a. bei älteren Kindern)

\section{Bildgebende Diagnostik}

- konventionelle Röntgenaufnahmen der Klavikula in a.-p.-Projektion 
Tab. 1 Klassifikation der Klavikulafrakturen nach Allmann.

\begin{tabular}{ll}
\hline Typ & Beschreibung \\
\hline Typ I & Fraktur im mittleren Drittel \\
\hline Typ II & Fraktur im lateralen Drittel \\
\hline Typ III & Fraktur im medialen Drittel
\end{tabular}

\section{Klassifikation}

(Tab. 1)

\section{Konservative Therapie}

Nicht dislozierte oder gering dislozierte Frakturen werden im Rucksack- oder Gilchrist-Verband behandelt. Die Konsolidationszeiten betragen - abhängig vom Alter - 2-3 Wochen. Sportfähigkeit ist i.d.R. nach 4-6 Wochen zu erwarten.

\section{Operative Therapie}

In Ausnahmefällen bei älteren Kindern mit entsprechend weiter Markhöhle kann eine intramedulläre Stabilisierung (TENS) erfolgen. In Einzelfällen ist eine Plattenosteosynthese zu diskutieren. Bei lateralen Frakturen kann mit resorbierbarer Kordel bzw. Zuggurtung stabilisiert werden.

\section{Proximale Oberarmfraktur}

Ca. $40 \%$ der Oberarmfrakturen sind im proximalen Segment, epiphysäre Frakturen sind extrem selten. In der Regel handelt es sich um Epiphysenlösungen (Typ Salter-Harris I oder II). Ursachen sind sowohl direkte als auch indirekte Traumen, selten Geburtstrauma (schwierige Diagnostik wegen fehlendem Knochenkern des Kopfes).

Die proximale Humerusepiphyse stellt sich im a.-p.- Röntgenbild zeltförmig dar, im seitlichen Bild konvex. Die Knochenkerne im Humeruskopf bilden sich erst im 1. Lebensjahr und verschmelzen zwischen dem 4. und 6. Lebensjahr. Durch das hohe Wachstumspotenzial der proximalen Humeruswachstumsfuge $(80 \%$ des Längenwachstums des Humerus) besteht ein hohes Korrekturpotenzial (Tab.2). Eine weitere Besonderheit ist die pathologische Fraktur bei juveniler Knochenzyste, die in diesem Segment besonders häufig auftritt. Wachstumsstörungen sind selten.
Tab. 2 Korrekturgrenzen am proximalen Oberarm in Abhängigkeit vom Alter.

\begin{tabular}{cl}
$<5$. LJ & $\begin{array}{l}\text { Frontalebene }<70^{\circ}, \\
\text { Seit-zu-Seit }<\text { Schaftbreite }\end{array}$ \\
\hline 5.-12. LJ & $\begin{array}{l}\text { Frontalebene }<50^{\circ}, \\
\text { Seit-zu-Seit }<1 / 2 \text { Schaftbreite }\end{array}$ \\
\hline$>12$. LJ & $\begin{array}{l}\text { Frontalebene }<30^{\circ}, \\
\text { Seit-zu-Seit }<1 / 2 \text { Schaftbreite }\end{array}$
\end{tabular}

Klinische Diagnostik

- schmerzhafte Bewegungseinschränkung und z.T. kompletter Funktionsverlust (Pseudoparalyse)

\section{Bildgebende Diagnostik}

- konventionelle Röntgenaufnahmen des Schultergelenks in 2 Ebenen, ggf. Ellenbogengelenk mit abbilden

- MRT bei fehlenden Knochenkernen oder schlecht zu beurteilenden Knochenkernen

\section{Klassifikation}

- AO-Klassifikation

\section{Konservative Therapie}

Bei insgesamt hohem Spontankorrekturpotenzial ist die proximale Humerusfraktur die Domäne der konservativen Therapie. Ruhigstellung im Gilchrist-Verband und Schmerzbehandlung, Konsolidierungszeit 3 Wochen, Krankengymnastik in der Regel nicht erforderlich. Sportfähigkeit ca. 3 Wochen nach Schmerzfreiheit.

\section{Operative Therapie}

Indikation zur Operation gegeben bei Abkippung bzw. stärkerer Fehlstellung (s.o.). Standardverfahren ist die geschlossene Reposition und Stabilisierung mit retrograden TENS (übungsstabil). Bei Weichteilinterposition (Bizepssehne) gegebenenfalls offene Reposition und Stabilisierung mit Kirschner-Drähten. Nachbehandlung frühfunktionell; Metallentfernung nach knöcherner Konsolidierung. Sportfähigkeit 3 Wochen nach Konsolidierung.

\section{Oberarmschaftfraktur}

Ca. 20\% der Oberarmfrakturen sind Schaftfrakturen. Meist direktes Trauma. Auch im Rahmen von Kindesmisshandlungen und pathologischen Frakturen bei Knochenzysten.
Tab. 3 Korrekturgrenzen am Oberarmschaft.

\begin{tabular}{ll}
\hline Ad latum & $<$ schaftbreit \\
\hline Achsfehlstellung & $<10^{\circ}$ \\
\hline Rotationsfehlstellung & $0^{\circ}$
\end{tabular}

Frakturen am Übergang zwischen mittlerem und distalem Schaftdrittel können zu Läsionen des N. radialis führen. Operative Revisonen des N. radialis sind selten notwendig (neurologische Verlaufskontrollen, Tab. 3).

\section{Klinische Diagnostik}

- schmerzhafte Bewegungseinschränkung und z.T. kompletter Funktionsverlust

- Schwellung

\section{Bildgebende Diagnostik}

- konventionelle Röntgenaufnahmen des Oberarms mit benachbarten Gelenken

- evtl. Sonografie

\section{Klassifikation}

- AO-Klassifikation

\section{Konservative Therapie}

Nicht dislozierte Schräg- und Spiralfrakturen (innerhalb der Korrekturgrenzen) werden konservativ versorgt. Ruhigstellung im Gilchrist-Verband (ggf. DesaultVerband, Brace) für 2-4 Wochen. Röntgenkontrollen nach 1 Woche und nach Abnahme des Verbands. Nach Konsolidierung Bewegungs- und Belastungsaufbau. Sportfähigkeit ca. 4 Wochen nach Konsolidierung der Fraktur.

\section{Operative Therapie}

Indikation zur Operation gegeben bei Dislokation außerhalb der Korrekturgrenzen (s.o.), offenen Frakturen, instabilen, schmerzhaften Frakturen. Relative Indikation ist die frühfunktionelle Behandlung nach operativer Stabilisierung. Standardverfahren geschlossene Reposition und Stabilisierung mit TENS (übungsstabil). Selten Fixateur externe. Röntgenkontrollen postoperativ und nach 4 Wochen. Metallentfernung nach 3-4 Monaten (TENS). Sportfähigkeit 4 Wochen nach Konsolidierung. 


\section{Suprakondyläre Humerusfraktur}

Häufigste Verletzung am kindlichen Ellenbogen. Typische Fraktur im Wachstumsalter (Gipfel im 5. LJ). Ursache ist meist ein Sturz auf den ausgestreckten Arm (Extensionsverletzung). Selten direktes Trauma durch Sturz auf den Ellenbogen (Flexionsverletzungen).

\section{Klinische Diagnostik}

- schmerzhafte Bewegungseinschränkung und Schwellung am Ellenbogengelenk

- sichtbare Fehlstellung

- Durchblutung, Motorik und Sensibilität peripher $\rightarrow$ Begleitverletzungen Nerven und Gefäße

\section{Bildgebende Diagnostik}

- konventionelle Röntgenaufnahmen des Ellenbogengelenks in 2 Ebenen (positives Fettpolsterzeichen)

- evtl. Sonografie, MRT

\section{Klassifikation}

- Epiphysiolysen (sehr selten) nach Salter-Harris

- Klassifikation nach Laer (Tab. 4)

\section{Konservative Therapie}

Nicht dislozierte Frakturen (Typ I) oder Epiphysiolysen werden konservativ versorgt. Ruhigstellung im Oberarmgips für 2-3 Wochen (bei kleinen Kindern Blount-Verband) für 2-3 Wochen. Röntgenkontrollen nach 1 Woche.

\section{Operative Therapie}

Indikation zur Operation gegeben bei dislozierten oder instabilen Epiphysiolysen und Frakturen Typ II-IV. Manche Autoren empfehlen bei Typ-II-Frakturen eine konservative Therapie mit geschlossener Reposition und Gipsanlage. Aus unserer Sicht ist die operative Stabilisierung des Repositionsergebnisses indiziert, um sekundäre Dislokationen zu vermeiden. Standardverfahren geschlossene Reposition und Stabilisierung mit gekreuzten Kirschner-Drähten (Abb. 1 ad). Offene Reposition selten notwendig. Alternative: deszendierende ESIN. Nachbehandlung im Gips für 3-4 Wochen. Röntgenkontrollen postoperativ und nach 4 Wochen. Metallentfernung nach 3-4 Monaten (ESIN). Sportfähigkeit 4 Wochen nach Konsolidierung.
Tab. 4 Klassifikation der suprakondylären Humerusfrakturen nach von Laer.

\begin{tabular}{ll} 
Typ & Beschreibung \\
\hline Typ I & nicht dislozierte Fraktur \\
\hline Typ II & Dislokation in sagittaler Ebene \\
\hline Typ III & $\begin{array}{l}\text { Dislokation in } 2 \text { Ebenen und } \\
\text { Rotationsfehler }\end{array}$ \\
\hline Typ IV & komplett dislozierte Fraktur
\end{tabular}

Rotationssporn ventral muss nach Reposition verschwunden sein.

\section{Komplikationen}

- Wachstumsstörungen (Cubitus varus/ valgus), sehr selten

- iatrogene Nervenläsionen (N. ulnaris)

- sekundäre Dislokation

\section{(Epi-)kondyläre Humerusfraktur}

Epikondyläre Frakturen sind selten. 90\% Epicondylus ulnaris. Sowohl indirektes als auch direktes Trauma. Häufiger sind transkondyläre Humerusfrakturen (zweithäufigste Ellenbogenverletzung). Meist liegt eine Fraktur des Condylus radialis vor (90\%), seltener Frakturen des Condylus ulnaris und Y-Frakturen. Altersgipfel der Condylus-radialis-Frakturen bei ca. 4-5 Jahren. Condylus ulnaris und Y-Frakturen später.

\section{Klinische Diagnostik}

- schmerzhafte Bewegungseinschränkung und Schwellung am Ellenbogengelenk

\section{Bildgebende Diagnostik}

- konventionelle Röntgenaufnahmen des Ellenbogengelenks in 2 Ebenen

- evtl. Sonografie, MRT

\section{Konservative Therapie}

Nicht dislozierte Frakturen (Spalt $<0,2 \mathrm{~cm}$ ) werden konservativ versorgt. Ruhigstellung im Oberarmgips für 3-4 Wochen. Röntgenkontrollen nach einer Woche und nach Gipsabnahme.

\section{Operative Therapie}

Indikation zur Operation gegeben bei dislozierten oder instabilen Frakturen. Standardverfahren offene Reposition und Stabilisierung mit Schraubenosteo- synthese. Nachbehandlung evtl. frühfunktionell oder im Gips für 3-4 Wochen. Röntgenkontrollen postoperativ und nach 4 Wochen. Metallentfernung nach 3-4 Monaten. Sportfähigkeit 4 Wochen nach Konsolidierung.

\section{Komplikationen}

- Pseudarthrosen des ulnaren Epikondylus (40\% nach konservativer Therapie, $10 \%$ nach OP)

- Nervenläsionen (N. ulnaris)

- sekundäre Dislokation

- stimulative Wachstumsstörungen

\section{Ellenbogenluxation}

Ellenbogenluxationen treten v.a. ab dem 12. Lebensjahr auf. Die Häufigkeit ist 3$6 \%$. Ursache ist meist ein Sturz auf die ausgestreckte Hand, in seltenen Fällen auch ein direktes Trauma im Rahmen eines Sturzes auf den dorsalen Ellenbogen.

\section{Klinische Diagnostik}

- Fehlstellung, Schmerzen und Bewegungseinschränkung

- Nach Reposition ist die klinische Prüfung und Beurteilung der Stabilität in Sedierung obligat.

- Kontrolle und Dokumentation von Durchblutung, Motorik und Sensibilität peripher

\section{Bildgebende Diagnostik}

- konventionelle Röntgenaufnahmen des Ellenbogengelenks in 2 Ebenen

- CT: zur Bilanzierung der ossären Begleitverletzungen

- MRT: zur Bilanzierung der ligamentären Begleitverletzungen

Häufig können ossäre Begleitverletzungen erst in der Röntgenkontrolle nach Reposition nachgewiesen werden.

\section{Klassifikation}

Ellenbogenluxationen werden nach der Richtung eingeteilt. Am häufigsten sind radiodorsale Luxationen. Seltener sind ulnare oder ventrale Luxationen.

\section{Konservative Therapie}

Isolierte Ellenbogenluxationen werden konservativ versorgt. Eine rasche Reposition nach Diagnosesicherung ist zwingend. Die Reposition erfolgt in Sedierung 


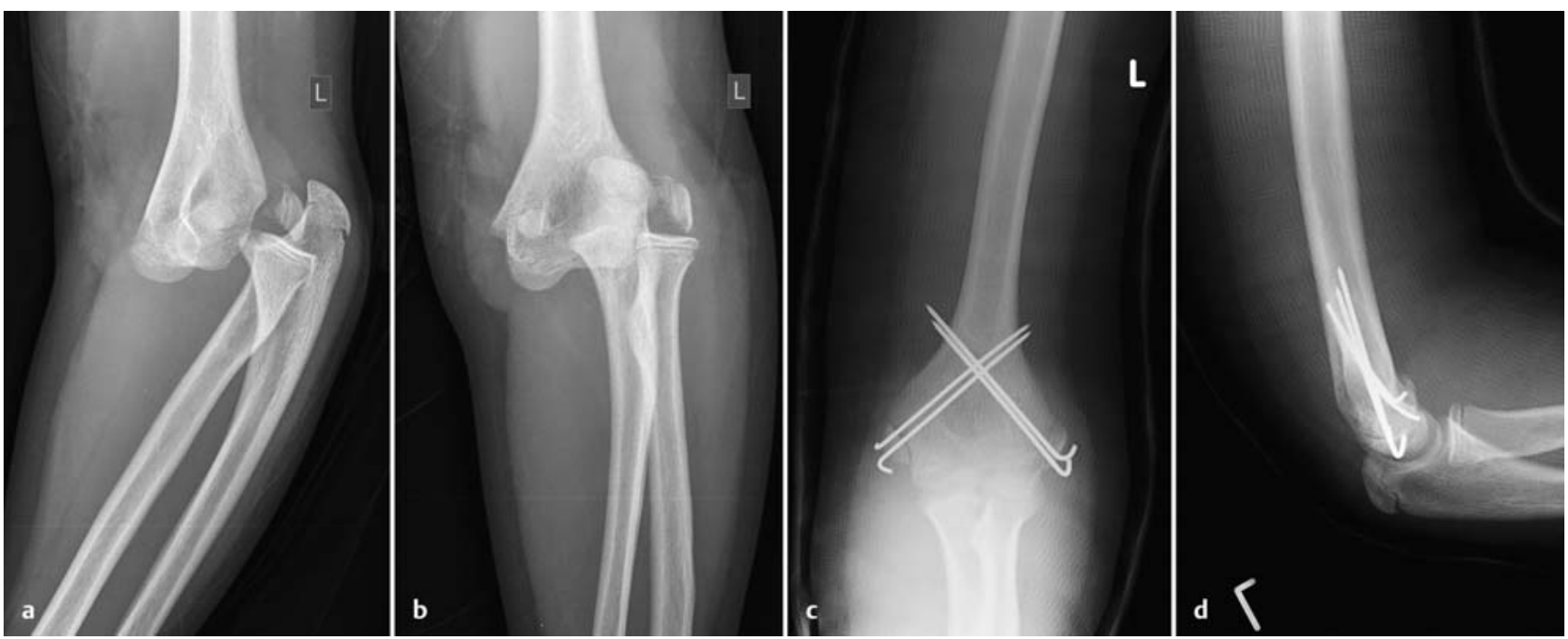

Abb. 1 a bis d 14-jähriger Junge mit einer suprakondylären Ellenbogenluxationsfraktur mit Abriss des Epicondylus radialis.
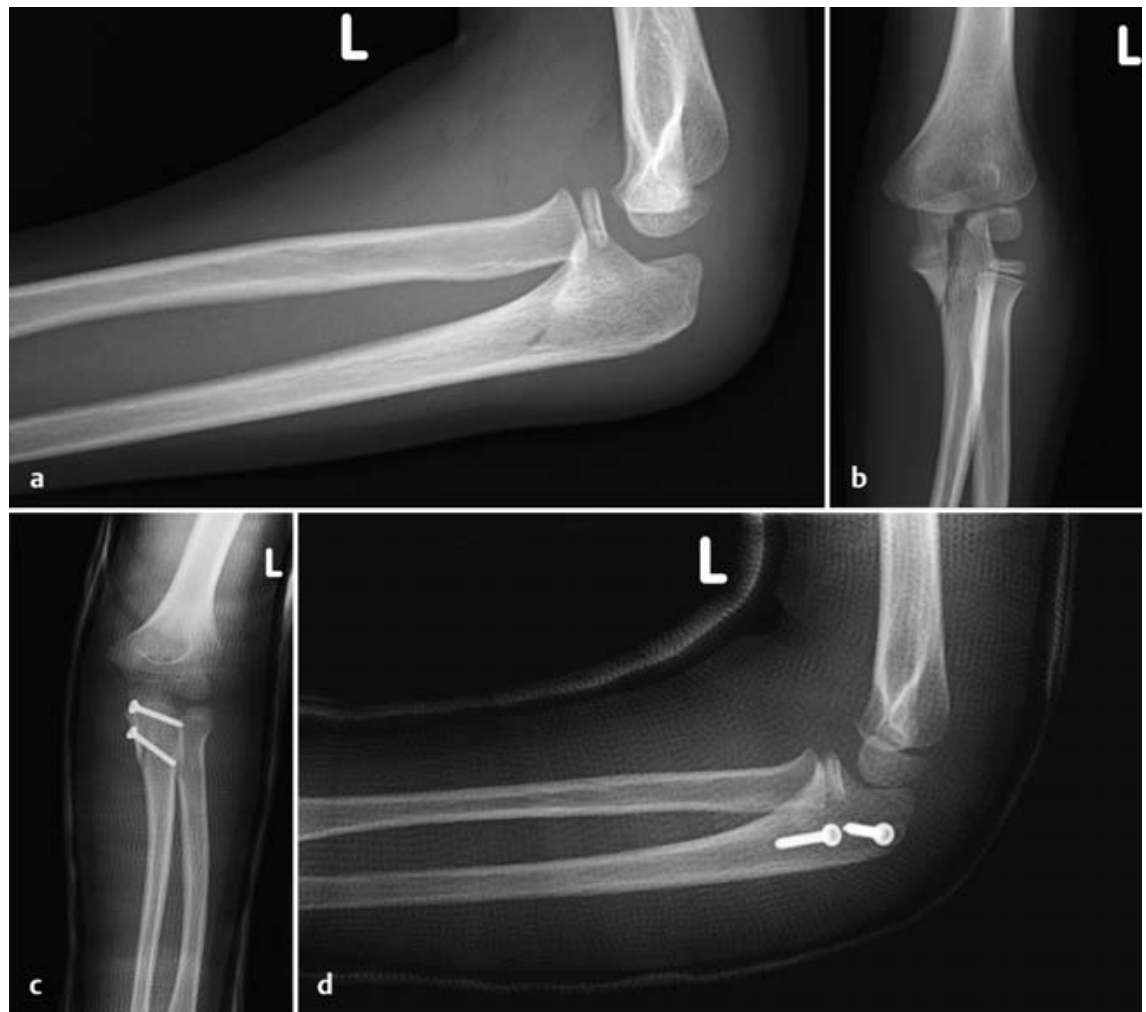

Abb. 2 a bis d 4-jähriges Mädchen mit Olekranon-Längsfraktur nach Sturz von einem Hochbett. Offene Reposition und Stabilisierung mit 2 2,7-mm-Zugschrauben.

oder Kurznarkose, ggf. unter Bildwandlerkontrolle. Nach Repositionsmanöver wird die Bandstabilität klinisch geprüft und eine Oberarmgipsschiene angelegt. Eine Röntgenkontrolle nach Reposition wird durchgeführt. Bei klinischem Verdacht auf eine ligamentäre Läsion kann diese mit einer MRT verifiziert werden. Nach Konsolidierung der Weichteile sollte der Gips zirkuliert werden. Insgesamt erfolgt eine Ruhigstellung für 2-3 Wo- chen. Anschließend kann funktionell nachbehandelt werden.

\section{Operative Therapie}

Ellenbogenluxationen mit Instabilität nach Reposition und Ellenbogenluxationen mit ossären Begleitverletzungen werden operativ versorgt. Ossäre Begleitverletzungen werden nach Reposition mit K-Drähten oder Kleinfragment- schrauben fixiert. Anschließend Ruhigstellung für ca. 3 Wochen im Oberarmgips.

\section{Olekranonfraktur}

Olekranonfrakturen im Wachstumsalter sind äußerst selten (Inzidenz < 1\%). Häufig in Kombination mit Radiusköpfchenluxationen oder proximalen Radiusfrakturen als Monteggia-Frakturen (cave: atypische Formen).

\section{Klinische Diagnostik}

- palpable Delle, Schmerzen und Bewegungseinschränkung

- Schwellung und Einblutung

\section{Bildgebende Diagnostik}

- konventionelle Röntgenaufnahmen des Ellenbogengelenks in 2 Ebenen

- Undislozierte Epiphysenfrakturen können nativradiologisch bei nicht sichtbaren Ossifikationskernen im Kleinkindesalter übersehen werden (ggf. Sonografie).

- Bei Kindern mit unspezifischen Beschwerden sollte das benachbarte Gelenk mituntersucht werden.

- Eine Monteggia-Läsion oder Monteggia-äquivalente Läsion muss immer ausgeschlossen werden.

\section{Konservative Therapie}

Nicht dislozierte (Frakturspalt $<2 \mathrm{~mm}$ ) Frakturen können konservativ therapiert werden. Therapie im Oberarmgips für 3 Wochen. Primär gespaltener Oberarmgips, nach Röntgenkontrolle (5.-7. Tag) Gipsschluss. 
Tipp: In der Praxis kann jeder nativradiologisch gut einsehbare Frakturspalt als mindestens $2 \mathrm{~mm}$ groß gewertet werden.

\section{Operative Therapie}

Dislozierte Frakturen werden offen reponiert und stabilisiert. Als Verfahren kommt die Zuggurtungsosteosynthese zur Anwendung. Bei Längs- und Schrägfrakturen evtl. auch Zugschrauben (Abb. 2a-d). Nach gesicherter Wundheilung kann eine funktionelle Nachbehandlung erfolgen. Nach Zugschraubenosteosynthese Ruhigstellung im Oberarmgips für 3-4 Wochen. Metallentfernung nach ca. 8 Wochen [7].

\section{Radiusköpfchen- und Radiushalsfrakturen}

Seltene Verletzung (ca. 5\% aller Ellenbogenfrakturen im Wachstumsalter). Ursache ist meist ein indirektes Trauma durch Sturz auf die dorsalflektierte Hand bei gestrecktem Ellenbogengelenk. Das Radiusköpfchen besteht im Wachstumsalter fast ausschließlich aus Knorpel. Daher im Wachstumsalter fast ausschließlich metaphysäre Frakturen am proximalen Radius. Meist Stauchungsfrakturen, seltener sind Epiphysiolysen.

Die Korrekturgrenze beträgt bis zu einem Alter von 10 Jahren $60^{\circ}$ Abkippung. Bei älteren Kindern reduziert sich diese Grenze auf $20^{\circ}$ Abkippung. Seitzu-Seit-Dislokationen werden nicht wesentlich korrigiert.

\section{Klinische Diagnostik}

- Schmerzen und Bewegungseinschränkung (v.a. Pronation und Supination)

- evtl. Druckschädigung des N. radialis

\section{Bildgebende Diagnostik}

- konventionelle Röntgenaufnahmen des Ellenbogengelenks in 2 Ebenen, Herausdrehen des Radiusköpfchens

- MRT (bei schwierig zu beurteilenden Röntgenbildern, fehlender Ossifikation des Radiusköpfchens)

- Gering dislozierte Frakturen können übersehen werden.

- Positives Fettpolsterzeichen oder Zeichen eines intraartikulären Ergusses können indirekte Frakturzeichen sein.
Tab. 5 Einteilung der diaphysären Unterarmfrakturen nach morphologischen Kriterien.

Grünholzfraktur, Wulstfraktur, Stauchungsfraktur

\begin{tabular}{l}
\hline offene Fraktur, geschlossene Fraktur \\
\hline Schräg-, Quer-, Torsion-, Trümmerfraktur \\
\hline komplette Unterarmfraktur, \\
isolierte Radius- oder Ulnafraktur \\
\hline stabile, instabile Fraktur
\end{tabular}

\section{Konservative Therapie}

Radiushalsfrakturen mit tolerabler Dislokation (s. Besonderheiten) und nicht dislozierte Radiusköpfchenfrakturen werden konservativ therapiert. Initial gespaltener Oberarmgips oder eine umgreifende Oberarmgipsschiene. Röntgenkontrolle nach 1 Woche. Insgesamt Ruhigstellung für 14 Tage. Im Anschluss kann frühfunktionell nachbehandelt werden.

\section{Operative Therapie}

Radiusköpfchenfrakturen mit Gelenkstufe und Radiushalsfrakturen mit entsprechender Dislokation werden operativ versorgt. Möglichst geschlossene Reposition und Stabilisierung mit TENS. Dislozierte Radiusköpfchenfrakturen mit Gelenkstufe müssen ggf. offen reponiert und mit einer Minifragmentschraube fixiert werden. Postoperative kurze Ruhigstellung (1-2 Wochen), anschließend funktionelle Therapie. Metallentfernung ca. 6 Wochen postoperativ [2].

Offene Repositionsmanöver führen häufig zu schlechten funktionellen Ergebnissen (ausgeprägte intraartikuläre Weichteilvernarbungen).

\section{Radiusköpfchensubluxation}

Syn.: Chassaignac-Luxation

Häufigste Verletzung des kindlichen Armes. Inzidenz 3,5\%. Ursache ist Längszug an der ausgestreckten Hand in Pronation.

\section{Klinische Diagnostik}

- Schonhaltung des Armes in Pronation

\section{Bildgebende Diagnostik}

- konventionelle Röntgenaufnahmen des Ellenbogengelenks in 2 Ebenen nach frustranem Repositionsversuch

\section{Konservative Therapie}

Immer konservative Therapie. Manuelle Reposition: Zug am Unterarm, dann Supination und Flexion im Ellenbogengelenk. Anschließend sofortige Beschwerdefreiheit. Bei 2 frustranen Repositionsversuchen Anlage einer Oberarmgipsschiene für 1-2 Tage. Anschließend häufig Beschwerdefreiheit (spontane Reposition).

\section{Diaphysäre Unterarmfraktur}

10-14\% aller Frakturen im Kindesalter sind diaphysäre Unterarmfrakturen. Ursache ist meist ein Sturz auf die ausgestreckte Hand. Meist im Rahmen von Unfällen im Freizeitsport, gefolgt von häuslichen Unfällen, selten Verkehrsunfällen. Seltener direkte Traumata (s. battered child). Meist treten sie im distalen Drittel auf.

Funktionell-anatomisch ist die Unterarmschaftfraktur als Gelenkfraktur zu sehen. Geringe Achsfehlstellungen können zu funktionellen Einschränkungen (Pronation/Supination) führen. Kein relevantes Remodelling diaphysär. Aus diesen Gründen empfehlen wir lediglich bei Kindern bis zum 5. Lebensjahr Achsfehlstellungen bis $20^{\circ} \mathrm{zu}$ tolerieren. Wachstumsstörungen nach diaphysären Unterarmfrakturen sind selten.

Achsfehlstellungen, die zu einer Einengung des interossären Raumes führen, dürfen nicht belassen werden.

\section{Klinische Diagnostik}

- Schonhaltung des Armes, Fehlstellung

Bildgebende Diagnostik

- konventionelle Röntgenaufnahmen des Unterarms mit benachbarten Gelenken

\section{Klassifikation \\ - nach Frakturtyp (Tab. 5) \\ - AO-Klassifikation}

\section{Konservative Therapie}

Stabile Frakturen (isolierte Fraktur eines Unterarmknochens, Grünholzfraktur) und Frakturen mit Achsabweichungen $<10^{\circ}$ (evtl. $<20^{\circ}$ ). Gegebenenfalls Reposition der Fraktur in Kurznarkose. Anlage eines zirkulären, gespaltenen Oberarmgipses. Nach Abschwellen der Weichteile 


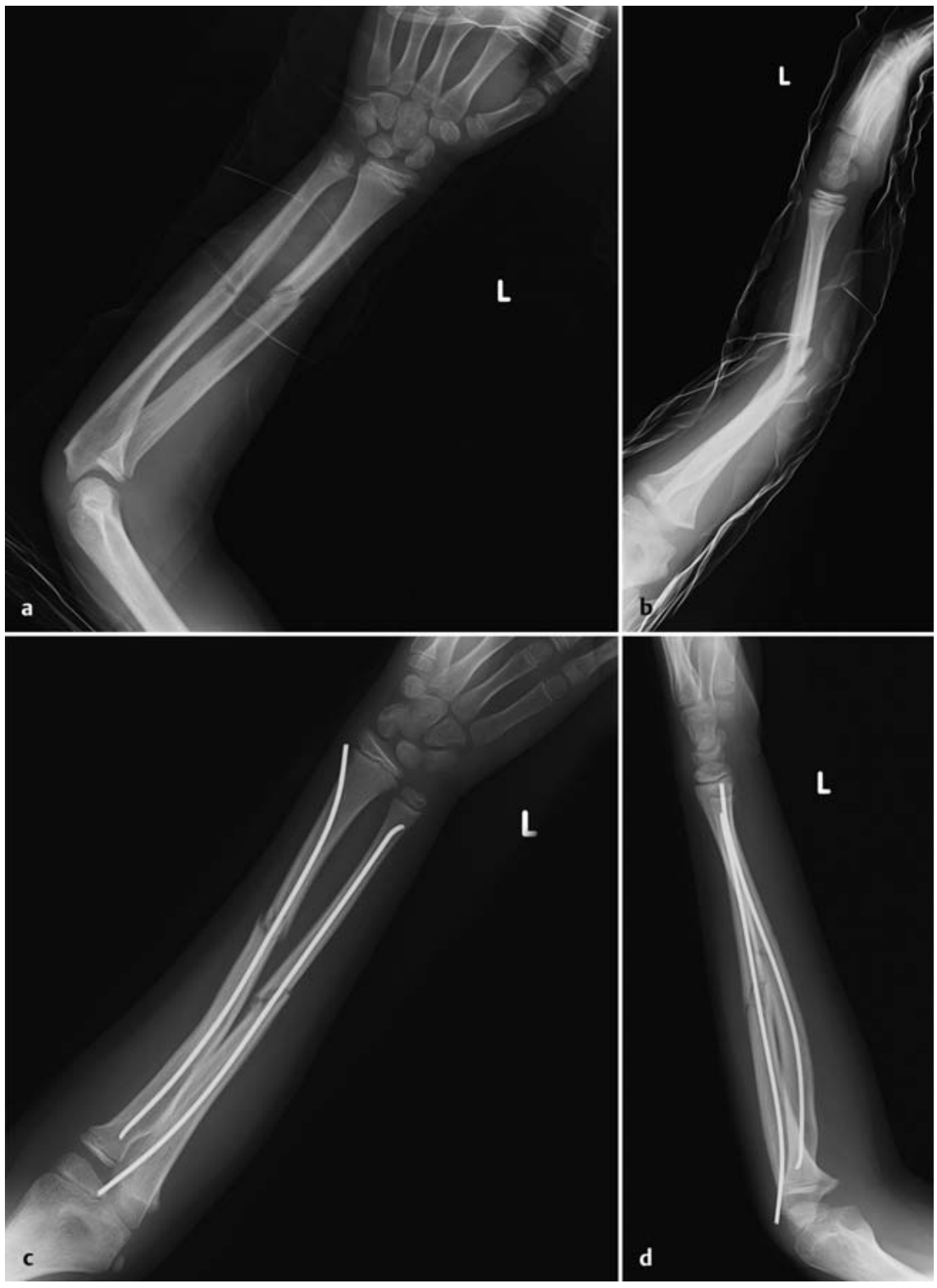

Abb. 3 a bis d 10-jähriger Junge mit diaphysärer Unterarmfraktur. Geschlossene Reposition und Stabilisierung mit TENS.

wird der Gips geschlossen. Insgesamt ist eine altersabhängige Ruhigstellung für 3-6 Wochen indiziert.

\section{Operative Therapie}

Instabile Frakturen (Fraktur beider Unterarmknochen) und Frakturen mit Achsabweichungen $>10^{\circ}$ werden operativ stabilisiert [4]. Offene Frakturen und begleitende Gefäß-, Nervenverletzungen, polytraumatisierte Kinder und sekundäre Dislokationen nach konservativer Therapie werden ebenfalls operativ versorgt. Mittel der Wahl ist die geschlossene Reposition und Osteosynthese mit TENS (Abb. $\mathbf{3} \mathbf{a}-\mathbf{d}$ ). Fixateur externe bei polytraumatisierten Kindern und deso-

\section{Besonderheiten}

Hohes Korrekturpotenzial (Nähe zur distalen Wachstumsfuge). Sowohl in der Frontal- als auch in der Sagittalebene werden Achsabweichungen bis $40^{\circ}$ bis zu einem Alter von 10 Jahren korrigiert. Nach dem 10. Lebensjahr beträgt die Korrekturgrenze 20-30 . Seit-zu-SeitFehlstellungen können bis zur Fehlstellung von $1 / 4$ Schaftbreite belassen werden. Ausgeprägtere Seit-zu-Seit-Fehlstellungen sollten reponiert werden, da sie zu einer länger dauernden Einschränkung der Supination und Pronation führen können. Rotationsfehlstellungen werden nicht wesentlich korrigiert und müssen reponiert werden. Es werden sowohl hemmende als auch stimulative Wachstumsstörungen nach distaler Unterarmfraktur beschrieben.

Häufige Repositionsmanöver und Refrakturen erhöhen das Risiko einer stimulativen Wachstumsstörung.

\section{Klinische Diagnostik}

- Schonhaltung des Armes, Fehlstellung, Paresen

\section{Bildgebende Diagnostik}

- konventionelle Röntgenaufnahmen des Unterarms mit benachbarten Gelenken in 2 Ebenen

\section{Klassifikation}

- AO-Klassifikation

\section{Konservative Therapie}

Stabile Frakturen und Frakturen mit tolerablen Achsabweichungen (s.o.). Gegebenenfalls Reposition der Fraktur in Kurznarkose. Anlage eines zirkulären, gespaltenen Oberarmgipses. Nach Abschwellen der Weichteile wird der Gips geschlossen. Insgesamt ist eine altersabhängige Ruhigstellung für 3-4 Wochen indiziert. Röntgenkontrollen nach 1 Woche und nach Gipsabnahme.

\section{Operative Therapie}

Instabile Frakturen (Fraktur beider Unterarmknochen) und Frakturen mit höhergradigen Achsabweichungen werden operativ stabilisiert. Offene Frakturen und begleitende Gefäß-, Nervenverletzungen, polytraumatisierte Kinder und sekundäre Dislokationen nach konservativer Therapie werden ebenfalls ope- 
rativ versorgt. Mittel der Wahl ist die geschlossene Reposition und Osteosynthese mit K-Draht (Abb.4a-d). Fixateur externe bei polytraumatisierten Kinder und desolater Weichteilsituation. Nachbehandlung im Unterarmgips für 4 Wochen. Anschließend Röntgenkontrolle und funktionelle Weiterbehandlung. Metallentfernung nach ca. 3-4 Monaten.

\section{Monteggia-Läsion}

Die Monteggia-Läsion ist eine Fraktur der Ulna kombiniert mit einer Radiusköpfchenluxation. Monteggia-Läsionen sind selten und machen ca. $1 \%$ der Frakturen im Wachstumsalter aus.

Monteggia-Läsionen werden häufig übersehen (30-50\%). Übersehene Monteggia-Läsion führen häufiger zu Wachstumsstörungen.

\section{Klinische Diagnostik}

- Schonhaltung des Armes, Fehlstellung, Paresen.

\section{Bildgebende Diagnostik}

- konventionelle Röntgenaufnahmen des Unterarms mit benachbarten $\mathrm{Ge}$ lenken in 2 Ebenen

\section{Konservative Therapie}

Selten konservative Therapie bei stabiler Ulnafraktur (Ausnahme).

\section{Operative Therapie}

Instabile Frakturen der Ulna. Mittel der Wahl ist die geschlossene Reposition der Ulna und Osteosynthese mit TENS. Bei metaphysären Frakturen auch K-Drahtoder Schraubenosteosynthese. Nach Reposition der Ulna meist spontane Reposition des Radiusköpfchens. Nachbehandlung im Oberarmgips für 2-4 Wochen. Anschließend Röntgenkontrolle und funktionelle Weiterbehandlung. Metallentfernung nach ca. 3-4 Monaten.

\section{Galeazzi-Läsion}

Als Galeazzi-Läsion bezeichnet man eine Fraktur des Radius kombiniert mit einer Luxation des distalen Radioulnargelenks. Sehr seltene Verletzung.

\section{Klinische Diagnostik}

- Schonhaltung des Armes, Fehlstellung, Paresen

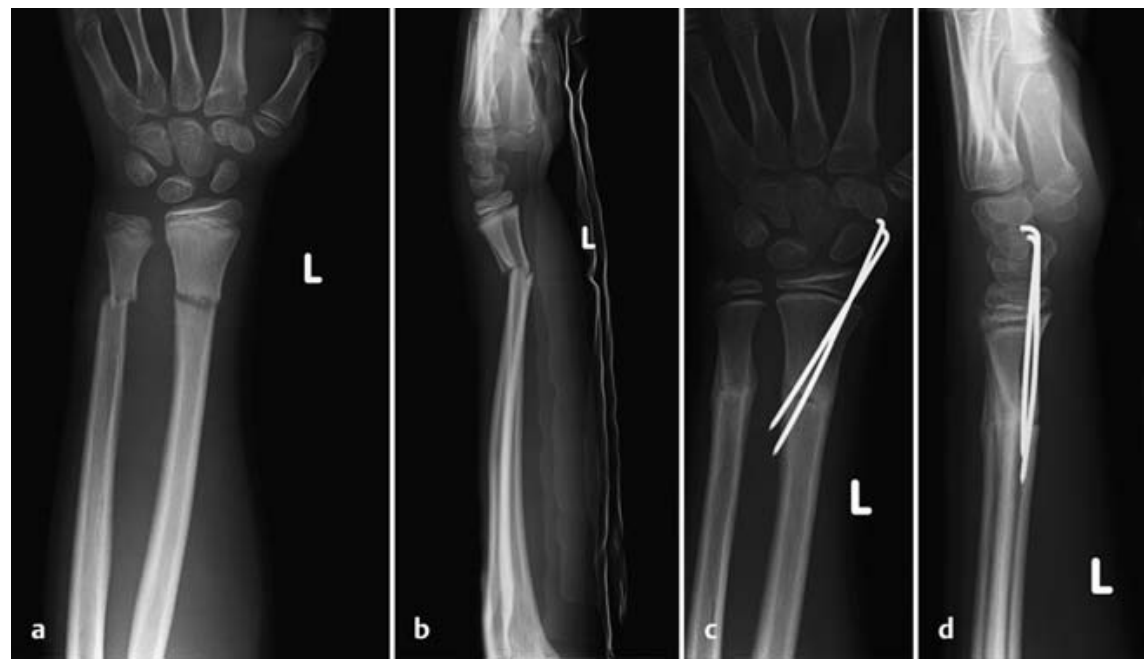

Abb. 4a bis d Distale metaphysäre Unterarmfraktur beim 12-jährigen Mädchen. Geschlossene Reposition und K-Draht-Osteosynthese.

\section{Bildgebende Diagnostik}

- konventionelle Röntgenaufnahmen des Unterarms mit benachbarten $\mathrm{Ge}$ lenken in 2 Ebenen

\section{Konservative Therapie}

Meist konservative Therapie möglich. Stabile Radiusfrakturen werden in Analgosedierung oder Vollnarkose geschlossen reponiert. Danach stellt sich die Ulna distal automatisch. Anschließend erfolgt die Anlage eines zirkulären, gespaltenen Oberarmgipses.

\section{Operative Therapie}

Instabile Radiusfrakturen werden geschlossen reponiert und mit einer intramedullären Schienung stabilisiert. In Ausnahmefällen ist eine offene Reposition und eine Plattenosteosynthese indiziert. Unabhängig von der Methode erfolgt eine Ruhigstellung im Oberarmgips für 4 Wochen. Der Gips wird nach Konsolidierung der Weichteile geschlossen. Röntgenkontrollen erfolgen nach Gipsschluss und nach Gipsabnahme.

\section{Handwurzel- und Mittelhandfrakturen}

Selten.

\section{Fingerfrakturen und -luxationen}

Häufigste Verletzungen der kindlichen Hand. Ursache Sturz auf die Hand.

\section{Klinische Diagnostik}

- Schmerzen und Schwellung, Schonhaltung
Bildgebende Diagnostik

- konventionelle Röntgenaufnahmen des Fingers in 2 Ebenen

Klassifikation

- Salter-Harris

\section{Konservative Therapie}

Frakturen innerhalb der Korrekturgrenzen. Gegebenenfalls Reposition in LA nach Oberst. Ruhigstellung mittels Gipsschiene in Intrinsic-plus-Stellung für 3-4 Wochen. Röntgenkontrollen nach einer Woche und nach Gipsabnahme.

\section{Operative Therapie}

Instabile und dislozierte (Gelenkstufe $>1 \mathrm{~mm}$ ) Frakturen werden operiert. Standardverfahren ist die geschlossene Reposition und K-Draht-Osteosynthese. Nachbehandlung mittels Gipsschiene in Intrinsic-plus-Stellung für 4-6 Wochen. Röntgenkontrollen nach 1 Woche und nach Gipsabnahme. Metallentfernung nach 4 Wochen. Sportfähigkeit nach 8 Wochen.

\section{Schlussfolgerung}

Frakturen im Bereich der oberen Extremität sind im Kindesalter häufige Verletzungen. Sofern sie zügig und korrekt diagnostiziert, kindgerecht und suffizient therapiert und adäquat nachbehandelt werden, haben sie meist eine gute Prognose. Kommt es zu Beteiligung der Wachstumsfugen, kann eine Wachstumsstörung (stimulative oder hemmende Wachstumsstörung) mit entspre- 
chenden Problemen resultieren. Die Behandler von Frakturen im Wachstumsalter müssen die Grenzen der Spontankorrektur kennen und respektieren.

\section{Literatur}

1 von Laer L. Frakturen und Luxationen im Wachstumsalter. 3. neubearb. Aufl. Stuttgart New York: Thieme; 2001

2 Schmittenbecher PP, Haevernick B, Herold A et al. Treatment decision, method of osteosynthesis and outcome in radial neck fractures in children: a multicenter study. J Pediatr Orthop 2005; 25: 45-50
${ }^{3}$ Schmittenbecher PP. Complications and errors in use of intramedullary nailing in shaft fractures in childhood. Kongressbd Dtsch Ges Chir Kongr 2001; 118: 435-437

${ }^{4}$ Schlickewei W, Salm R. Indications for intramedullary stabilization of shaft fractures in childhood. What is reliable, what is assumption? Kongressbd Dtsch Ges Chir Kongr 2001; 118: 431-434

5 Oberle M, Schlickewei W. Elastic-stable-intramedullary nailing for forearm fractures in children using TENS. Unfallchirurg 2005; 108: 235-237

${ }^{6}$ Schlickewei W, Oberle M. Forearm fractures in children. Unfallchirurg 2005; 108: 223-232

7 Weise K, Schwab E, Scheufele TM. Elbow injuries in childhood. Unfallchirurg 1997; 100: 255-269
Dr. med. Mike Oberle

Oberarzt

Prof. Dr. med. Wolfgang Schlickewei

Chefarzt

Abteilung Unfall- und Wiederherstellungschirurgie, Kindertraumatologie Regionalverbund Kirchlicher Krankenhäuser (RkK) St. Josefskrankenhaus

Sautierstraße 1

79104 Freiburg

mike.oberle@t-online.de wolfgang.schlickewei@rkk-sjk.de 